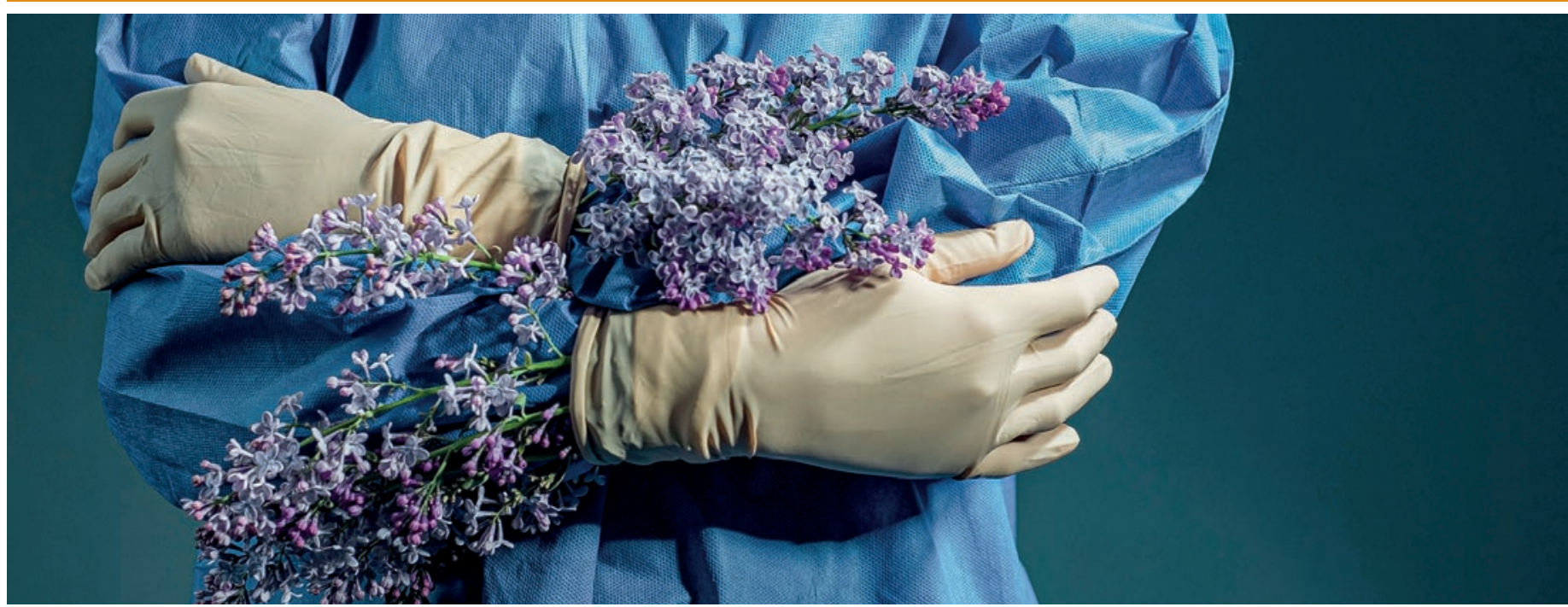

\title{
Nachhaltiges Gesundheitswesen
}

\section{Christian Abshagen}

Dr. med., DTM\&H, MBA, Leiter Fachstelle Nachhaltigkeit, Universitätsspital Basel, Wissenschaftlicher Mitarbeiter Institut für Ecopreneurship, Hochschule für Life Sciences, Fachhochschule Nordwestschweiz (FHNW)

Immer mehr wissenschaftliche Allianzen rücken die Verflechtung von Gesundheit und Umwelt in den Fokus. In direkter Beziehung dazu unternehmen praxisorientierte Initiativen Anstrengungen, die Auswirkungen des Gesundheitssektors auf die Umwelt zu reduzieren.

Das Gesundheitswesen ist global für ca. 4,4\% der Treibhausgasemissionen verantwortlich. Die USA, China und Europa allein verursachen etwa die Hälfte dieser Emissionen [1]. Vor diesem Hintergrund ist die im Oktober 2020 durch den National Health Service (NHS) des Vereinigten Königreichs veröffentlichte Erklärung [2], als erstes Gesundheitssystem der Welt klimaneutral werden $\mathrm{zu}$ wollen, ein folgerichtiger Schritt. In einem umfassenden Bericht definiert der NHS Reduktionspfade und Enddaten und übernimmt die Verantwortung für Treibhausgasemissionen bis in die vor- und nachgelagerten Prozesse von Produktion, Entsorgung und Mobilität.

Dieses Statement darf nicht darüber hinwegtäuschen, dass die Umsetzung anspruchsvoll wird und dass alle Gesundheitssysteme diesem Weg folgen sollten.

\section{Auswirkungen des Gesundheitssystems auf die Umwelt}

Mindestens genauso wichtig ist es zu realisieren, dass die Einflüsse des Gesundheitssektors auf die Umwelt weit über dessen Treibhausgasemissionen hinausgehen. Beispielhaft genannt seien:
- Infrastruktur und Energie: Alte Gebäude bedürfen energetischer Sanierungen; die Auswahl der Baumaterialien bleibt über den Lebenszyklus umweltrelevant. Hohe Energieverbräuche von Bereichen wie Klimatisierung und Radiologie gilt es zu minimieren.

- Verpflegung: Für Patientinnen und Patienten sowie für die Mitarbeiterschaft werden grosse Mengen an Lebensmitteln beschafft, verbraucht oder landen im Abfall. Deren ökologischer Fussabdruck ist durch Landverbrauch, Energie- und Pestizideinsatz, Verbreitung von Monokulturen, den Verlust an Biodiversität und anderes mehr besonders bedeutend.

- Medikamente: Die Anzahl in der Schweiz zugelassener und verbrauchter Medikamente steigt infolge technologischen Fortschritts und demographischer Entwicklung. Mikroverunreinigungen in Gewässern stellen eine wachsende Herausforderung dar.

- Chemikalien: Ihr Einsatz ist in pharmazeutischer Forschung, Entwicklung und Produktion sowie in klinischer Routine (Labormedizin, Pathologie) und für Reinigung und Desinfektion unerlässlich - mit oftmals nachteiligen gesundheitlichen und ökologischen Effekten. 
- Einwegmaterialien und Abfälle: Der Einsatz von Einweg-Medizinprodukten nimmt weiter zu. Neben Unmengen von Plastik und Kunststoffen werden selbst hochwertige Ressourcen wie Metalle unzureichend in geschlossene Kreisläufe gebracht, womit wertvolle Rohstoffe verloren gehen.

\section{Beziehung von Umwelt und menschlicher Gesundheit}

Noch wesentlich vielschichtiger als die Einwirkungen des Gesundheitswesens auf die Umwelt sind die Verflechtungen von Umweltzuständen und menschlicher Gesundheit. Vergleichsweise gut erforscht und verstanden sind direkte Effekte, welche auf Basis von Dosis-Wirkungs-Beziehungen und durch epidemiologische Studien greifbar werden. Asbest und Mesotheliom, Feinstaubkonzentration und pulmonale Erkrankungen, Lärmbelastung und kardiovaskuläre Mortalität sind bekannte Beispiele. In Zeiten der Globalisierung, von Klimawandel und Biodiversitätsverlust jedoch werden die Wechselwirkungen komplexer, langfristiger und teilweise verschärft. Zoonosen werden durch Veränderungen der Landnutzung und Zerstörung natürlicher Habitate begünstigt, globaler Handel und Mobilität erleichtern deren Verbreitung. Die Effekte einer Erwärmung auf die Verbreitung von vektor-übertragenen Krankheiten oder den Nährstoffgehalt von pflanzlichen Nahrungsmitteln stehen exemplarisch für die Verwobenheit von Klimawandel, Biodiversität und menschlicher Gesundheit.

Durch Umweltveränderungen ausgelöste oder wahrscheinlicher werdende Naturkatastrophen, Migration und bewaffnete Konflikte verursachen für sich selbst sowohl direkte als auch indirekte Gesundheitsschäden. Zu letzteren gehören Auswirkungen von Umweltveränderungen auf die psychische Gesundheit, die noch unzureichend verstanden werden.

Neben den Herausforderungen können sich aber auch sogenannte Co-Benefits ergeben in der Ernährung, der Mobilität oder der psychischen Gesundheit, bei denen eine Besserstellung der Umwelt mit Zugewinnen für die Gesundheit einhergeht.
In diesem Kontext rücken sogenannte Ökosystemleistungen in den Fokus: Die Umwelt stellt beispielsweise pharmazeutische Wirkstoffe bereit, reguliert und mitigiert Schadstoffwirkungen und bietet Genesungs- und Erholungswert.

\section{Aktuelle internationale Entwicklungen}

An den Schnittstellen von Public Health zu Infektionskrankheiten und Veterinärmedizin sind seit etlichen Jahren akademische Ansätze etabliert, die eine enge Beziehung zwischen Mensch und Umwelt postulieren und beforschen. Am bekanntesten sind der OneHealth-Ansatz (organisiert z.B. in der OneHealth platform [3]) und die EcoHealth Alliance [4].

Wesentlich jünger, weiter gefasst und sich global rasch entwickelnd ist die 2016 durch die Rockefeller Foundation und den Lancet ins Leben gerufene und an der

\section{Das Gesundheitswesen der Schweiz steht}

in puncto Nachhaltigkeit erst am Anfang.

Harvard University beheimatete Planetary Health Alliance [5]. Diese befasst sich transdisziplinär und ganzheitlich mit dem Gesundheitszustand der menschlichen Zivilisation und der sie umgebenden Umwelt, von der sie abhängt. Zu ihren Mitgliedern zählen neben den zwei zuvor genannten Initiativen inzwischen weltweit mehr als 240 Universitäten, akademische Einrichtungen und Organisationen.

Eine vergleichbare, aber praxisorientiertere Entwicklung lässt sich auch aufseiten von ökologischer Nachhaltigkeit im Gesundheitswesen (Sustainable Healthcare) beobachten. Der eingangs zitierte NHS hat ein Center for Sustainable Healthcare [6] etabliert, das den Wandel des NHS zu einem nachhaltigeren Gesundheitswesen gestaltet. In den skandinavischen Ländern existiert mit dem Nordic Center for Sustainable Healthcare [7] ein Pendant. Unbedingt zu erwähnen ist die global tätige Non-ProfitOrganisation Healthcare without harm [8], deren Mission es ist, das Gesundheitswesen zu ökologischer Nachhaltigkeit zu transformieren und die durch ihre ca. 1500 Mitglieder weltweit etwa 43000 Spitäler und Gesundheitszentren repräsentiert.

\section{Welche Lücken gibt es in der Schweiz?}

Trotz dieser dynamischen Entwicklungen steht das Schweizer Gesundheitswesen erst am Anfang. Herausforderungen gilt es, auf verschiedenen Ebenen zu adressieren:

- Studentische Ausbildung: Bislang wird erst in einer Minderheit der Medizinstudiengänge zu gesundheitlichen Auswirkungen von Umweltveränderun- 
gen und ökologischer Nachhaltigkeit gelehrt. Die Association for Medical Education in Europe (AMEE) hat dieses Defizit erkannt und fordert in ihrem Konsensuspapier, dass Ausbildung in ökologisch nachhaltiger Gesundheitsversorgung und Planetary Health als transversale Themen in die Curricula integriert werden sollen [9].

- Weiterbildung: Für die heute im Schweizer Gesundheitswesen Berufstätigen gibt es kaum Angebote, um sich zu den genannten Themenkomplexen fortzubilden. Da etliche Herausforderungen nur transdisziplinär, berufsgruppenübergreifend und durch enge Zusammenarbeit der verschiedenen Stakeholder gelöst werden können, sollten solche Angebote idealerweise breit zugänglich sein.

- Forschung: Es existiert eine Vielzahl an Forschungslücken, welche auf verschiedenen Ebenen und durch die Kooperation akademischer Institutionen mit der Privatwirtschaft geschlossen werden können. Das Forschungskonzept Umwelt 2021 bis 2024 des Bundesamtes für Umwelt identifiziert «Gesundheit und Umwelt» als eines von fünf zentralen Forschungsthemen, das ressortübergreifend für die Bundesstellen von hohem Interesse ist [10].

- Praxis: Funktionen in Spitälern und Gesundheitseinrichtungen, welche sich mit der strategischen Ausrichtung auf und der Transformation hin zu ökologischer Nachhaltigkeit befassen, sind - im Gegensatz zur privaten Wirtschaft - noch viel zu wenig etabliert.

- Wissenschaftliche Zusammenschlüsse wie die Planetary Health Alliance erforschen die Verflechtungen von menschlicher Gesundheit und globalen Umweltveränderungen.

Dr. med.

Christian Abshagen,

Institut für Ecopreneurship Hochschule für Life Sciences Fachhochschule Nordwestschweiz

Hofackerstrasse 30

CH-4132 Muttenz

christian.abshagen[at]

fhnw.ch ches zum heutigen Stand nicht nachhaltig ist.

- Die Schweiz steht im Bereich der Sustainable Healthcare noch mehreren Herausforderungen gegenüber, denen durch eine Intensivierung der Forschungsaktivitäten und einen Ausbau der Aus- und Fortbildungsangebote begegnet werden muss.

\section{Das Wichtigste in Kürze}

Die Zeit war nie reifer als in einer Covid-Welt, diese Herausforderungen gemeinsam anzugehen. Der Zusammenschluss der nationalen Akademien der Wissenschaften der EU-Staaten, Norwegens und der Schweiz (EASAC) hat dies jüngst so formuliert: «Addressing climate change, loss of biodiversity and resource depletion cannot avoid addressing the underlying drivers of unsustainable consumption and production patterns in society's core systems» [11].

\section{Literatur}

1 Health Care Without Harm. Health care climate footprint report. 2019. https://noharm-uscanada.org/ClimateFootprintReport

2 Delivering a 'Net Zero' National Health Service. NHS England and NHS Improvement. October 2020

3 https://onehealthplatform.com/

4 https://www.ecohealthalliance.org/

5 https://www.planetaryhealthalliance.org/planetary-health

$6 \mathrm{https://sustainablehealthcare.org.uk/}$

7 https://nordicshc.org/

8 https://noharm.org/

9 Shaw E, Walpole S, McLean M, Alvarez-Nieto C, Barna S, et al. AMEE Consensus Statement: Planetary health and education for sustainable healthcare. Medical Teacher, 2021.

10 Forschungskonzept Umwelt 2021-2024. Forschungsbereiche und prioritäre Forschungsthemen. Bundesamt für Umwelt (BAFU, Hrsg.), Bern. Umwelt-Info. 2020;2009:82.

11 Towards a sustainable future: transformative change and postCOVID-19 priorities. A perspective by EASAC's Environment Programme. European Academies Science Advisory Council (easac). perspective. October 2020, ISBN: 978-3-8047-4199-7.

Bildnachweis

Oleksii Donenko| Dreamstime.com

\section{L'essentiel en bref}

- Des alliances scientifiques telles que la Planetary Health Alliance explorent les interconnexions entre la santé humaine et les changements environnementaux globaux.

- L'accent est également mis sur l'impact considérable du système de santé lui-même sur l'environnement, système qui n'est pas durable à l'heure actuelle.
- La Suisse se trouve face à plusieurs défis en matière de système de santé durable (Sustainable Healthcare), qu'il convient de relever en intensifiant les activités de recherche et en développant les possibilités d'éducation et de formation. 